\title{
Role of probiotics and nutrition in the management of chronic inflammatory bowel disease in children
}

Seng Hock Quak, mBBS, MMed

I

$\mathrm{n}$ recent years, there has been a great increase in the range of chronic inflammatory conditions in developed countries. Singapore is no exception. In the early 1970s, inflammatory bowel disease was almost unheard of among the paediatric population in Singapore. Over the last few decades, however, there has been a steady rise in the number of Singaporean children suffering from either ulcerative colitis or Crohn's disease.

A postulated reason for this recent increase is an altered immune response to microorganisms in children. This is related to the so-called 'hygiene hypothesis', a theory, which for a number of years, had focused on allergies. However, recent evidence has shown that early childhood infection does not protect children from allergies. Thus, the Th1/Th2 imbalance hypothesis is unlikely to be the only explanation. In fact, simultaneous increases in both Th1 and Th17 responses have been observed in chronic inflammatory diseases. ${ }^{(1)}$ Hence, the imbalance between immunoregulatory and effector mechanisms is a more likely explanation. ${ }^{(2)}$

The number of bacteria living in the human body far outnumbers the number of human cells. In the human gut alone, there is an estimate of about 10 to 100 trillion microbes. Through the evolution of human beings, we have become dependent on the presence of these commensal organisms. They are our 'old friends', and we are in a state of evolved dependence on this wide range of microorganisms, with which we co-evolve. The microbiota of city-dwelling children differs dramatically from that of rural-dwelling children. Compared to children living in developed countries, rural children have cellulose- and xylan-degrading microorganisms in their gut flora. These microorganisms carry out hydrolysis, a process that is responsible for the production of short-chain fatty acids, which have an antiinflammatory effect on the gut. Microorganisms within the gut affect the intestinal immune system, provide key nutrients and can modify energy metabolism.

The main medications used in the treatment of inflammatory bowel disease are corticosteroids and immunosuppressants, both of which have inherent side effects and complications. Most parents are concerned about the untoward effect of corticosteroids on children and seek alternative treatments. The use of probiotics is an attractive alternative in view of the hypothesis that altered bacterial flora may be a pathogenic factor. Although probiotics are well tolerated and considered harmless, the quality of studies done and the health claims made are widely variable. While studies on adults have shown the efficacy of probiotics in the management of pouchitis, similar studies on children are not available. In a recent study on the costeffectiveness of adjunct VSL\#3 therapy versus standard medical therapy in paediatric ulcerative colitis, Park et al showed that the quality of life was only marginally increased for medical therapy supplemented with VSL\#3. ${ }^{(3)}$ Another recent study on probiotics suggested that high-dose probiotics may be beneficial in acute paediatric ulcerative colitis exacerbation and maintenance of remission. ${ }^{(4)}$ However, the role of probiotics in Crohn's disease is less promising, and better quality trials as well as laboratory investigations on its mechanisms are needed to shed light on who will respond and which strains to use. ${ }^{(5,6)}$

Faecal microbiota transplantation, another alternative treatment for inflammatory bowel disease, has been the focus of research in recent years. However, the current evidence on its efficacy is weak and limited. The available data suggest that faecal microbiota transplantation has the potential to be an effective and safe treatment, particularly when the result of standard treatment is not satisfactory. ${ }^{(7-10)}$ Another nonconventional medical treatment is the nutritional therapy. Nutrition is implicated in every aspect of inflammatory bowel disease, from pathogenesis to treatment management. Although various dietary factors have been suggested to play a role in its pathogenesis, evidence for a major dietary factor is limited. ${ }^{(11)}$ In an appropriate environment, the onset of disease in a genetically predisposed individual may be triggered by the presence or absence of specific dietary components. A high caloric diet, high consumption of refined sugar and regular intake of processed fat may be risk factors. The consumption of various types of fat has been associated with increased risk, with high intake of n6-polyunsaturated fatty acids and low intake of n3-polyunsaturated fatty acids being linked to an elevated risk of chronic inflammatory bowel disease. On the other hand, a diet rich in dietary fibre and fruits appears to be protective. Based on epidemiological data, high consumption of dairy products is also a possible risk factor.

In children with inflammatory bowel disease, particularly Crohn's disease, malnutrition is a common feature. ${ }^{(12)}$ There are several possible reasons for the poor nutritional status of the children. Nausea and food aversion - symptoms of the disease - may cause affected children to have suboptimal nutritional intake, whereas diarrhoea and fever, the other symptoms of the disease, are associated with increased energy and nutrient loss. Another feature of inflammatory bowel disease is poor nutrient absorption, along with increased energy utilisation and altered metabolism. Abnormal anthropometric data, particularly weight

Correspondence: Dr Quak Seng Hock, Professor and Senior Consultant, Department of Paediatrics, Yong Loo Lin School of Medicine, National University of Singapore, 21 Lower Kent Ridge Road, Singapore 119007.senghock_quak@nuhs.edu.sg 
and height percentiles, are also commonly seen in children with inflammatory bowel disease. Faltering linear growth is frequently observed in children with the disease, and about a quarter of patients with Crohn's disease present with faltering growth velocity. Although the nutritional status of affected children can be rehabilitated with the appropriate treatment, they may still not achieve their genetic potential in terms of their final adult height.

With regard to the induction of remission, enteral nutrition has been used successfully in paediatric Crohn's disease. The rationale of using enteral feeds is 'gut rest', as enteral feeds consist of digested proteins, fat and carbohydrates, which require less digestive activities in the intestine and are more readily absorbed. Since enteral feeds spend less time in the intestine, the mucosal lining has less contact with possible antigens in the diet, thereby reducing the possibility of an inflammatory reaction. Enteral feeds are also postulated to work via modulation of the intestinal microflora. In a recent study, 11 out of 18 children with Crohn's disease were found to respond positively to exclusive enteral nutrition. These children showed decreased levels of proinflammatory acetic acid and increased concentrations of anti-inflammatory butyric and valeric acids. This supports the hypothesis that exclusive enteral nutrition has an anti-inflammatory effect in children with Crohn's disease, ${ }^{(13)}$ although the mode of action in suppressing mucosal inflammation is still not fully understood.

Enteral nutrition is usually used exclusively for two to three months, after which oral intake may be gradually introduced. The main limiting factor of this treatment is compliance, as exclusive enteral feeding for such a long period of time is almost impossible to implement, particularly in school-going children. In the Department of Paediatrics at National University Hospital, eight children who were newly diagnosed with Crohn's diseases were recently put on enteral nutrition therapy. Preliminary data suggest that eight weeks of enteral nutrition is associated with favourable outcome. ${ }^{(14)}$ The response is similar to the results reported in a study conducted in the Netherlands. ${ }^{(15)}$

In the adult population, concomitant use of enteral nutrition therapy is associated with sustained response to infliximab. ${ }^{16)}$ No similar study is, however, available in children. While the short-term effects of exclusive enteral nutrition for the induction of remission in children with Crohn's disease are promising, the long-term benefits are less clear. Lambart et al ${ }^{(17)}$ retrospectively reviewed the outcomes for up to 24 months following exclusive enteral feeding in 31 children compared to 26 children treated with corticosteroids. Of the 31 children treated with enteral nutrition therapy, 26 entered remission. They exhibited lower Crohn's disease activity index scores at six months and received lower cumulative doses of steroids over the study period. ${ }^{(17)}$ More long-term, well-powered studies are required to accurately assess the usefulness of nutrition in the induction and maintenance of remission.

\section{REFERENCES}

1. Himmel ME, Yao Y, Orban PC, Steiner TS, Levings MK. Regulatory T-cell therapy for inflammatory bowel disease: more questions than answers. Immunology 2012; 136:115-22.

2. Hardenberg G, Steiner TS, Levings MK. Environmental influences on T regulatory cells in inflammatory bowel disease. Semin Immunol 2011; 23:130-8.

3. Park KT, Perez F, Tsai R, et al. Cost-effectiveness analysis of adjunct $\mathrm{VSL} \# 3$ therapy versus standard medical therapy in pediatric ulcerative colitis. J Pediatr Gastroenterol Nutr 2011; 53:489-96.

4. Miele E, Pascarella F, Giannetti E, et al. Effect of a probiotic preparation (VSL\#3) on induction and maintenance of remission in children with ulcerative colitis. Am J Gastroenterol 2009; 104:437-43.

5. Jonkers D, Penders J, Masclee A, Pierik M. Probiotics in the management of inflammatory bowel disease: a systemic review of intervention studies in adult patients. Drugs 2012; 72:803-23.

6. Whelan K, Quigley EM. Probiotics in the management of irritable bowel syndrome and inflammatory bowel disease. Curr Opin Gastroenterol 2013; 29:184-9.

7. Meijer BJ, Dieleman LA. Probiotics in the treatment of human inflammatory bowel diseases: update 2011. J Clin Gastroenterol 2011; 45: S139-44.

8. Damman CJ, Miller SI, Surawicz CM, Zisman TL. The microbiome and inflammatory bowel disease: is there a therapeutic role for fecal microbiota transplantation? Am J Gastroenterol 2012; 107:1452-9.

9. Anderson JL, Edney RJ, Whelan K. Systematic review: faecal microbiota transplantation in the management of inflammatory bowel disease. Aliment Pharmacol Ther 2012; 36:503-16.

10. Aroniadis OC, Brandt LJ. Fecal microbiota transplantation: past, present and future. Curr Opin Gastroenterol 2013; 29:79-84.

11. Gentschew L, Ferguson LR. Role of nutrition and microbiota in susceptibility to inflammatory bowel diseases. Mol Nutr Food Res 2012; 56:524-35.

12. Gerasimidis K, McGrogan P, Edwards CA. The aetiology and impact of malnutrition in paediatric inflammatory bowel disease. J Hum Nutr Diet $2011 ; 24: 313-26$.

13. Tjellstrom BO, Hogberg L, Stenhammar L, et al. Effect of exclusive enteral nutrition on gut microflora function in children with Crohn's disease. Scand J Gastroenterol 2012; 47:1454-9.

14. Ong F, Gracia MA, Aw M, Quak SH. The Use of Exclusive Enteral Feeding as Primary Therapy for Crohn's Disease in Singaporean Children and Adolescents (poster). 4th World Congress of Pediatric Gastroenterology, Hepatology and Nutrition 2012; PP15046.

15. de Bie C, Kindermann A, Escher J. Use of exclusive enteral nutrition in paediatric Crohn's disease in The Netherlands. J Crohns Colitis 2013; 7:263-70.

16. Sazuka S, Katsuno T, Nakagawa T, et al. Concomitant use of enteral nutrition therapy is associated with sustained response to infliximab in patients with Crohn's disease. Eur J Clin Nutr 2012; 66:1219-23.

17. Lambart B, Lemberg DA, Leach ST, Day AS. Longer-term Outcomes of Nutritional Management of Crohn's Disease in Children. Dig Dis Sci 2012; 57:2171-7. 